\title{
Polygenic risk for coronary artery disease in the Scottish and English population
}

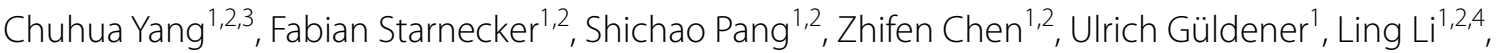 \\ Matthias Heinig ${ }^{4,5}$ and Heribert Schunkert 1,2,36*
}

\begin{abstract}
Background: Epidemiological studies have repeatedly observed a markedly higher risk for coronary artery disease (CAD) in Scotland as compared to England. Up to now, it is unclear whether environmental or genetic factors might explain this phenomenon.

Methods: Using UK Biobank (UKB) data, we assessed CAD risk, based on the Framingham risk score (FRS) and common genetic variants, to explore the respective contribution to CAD prevalence in Scotland $(n=31,963)$ and England $(n=317,889)$. We calculated FRS based on sex, age, body mass index (BMI), total cholesterol (TC), high density lipoprotein cholesterol (HDL-C), systolic blood pressure (SBP), antihypertensive medication, smoking status, and diabetes. We determined the allele frequency of published genome-wide significant risk CAD alleles and a weighted genetic risk score (WGRS) for quantifying genetic CAD risk.

Results: Prevalence of CAD was 16\% higher in Scotland as compared to England (8.98\% vs. $7.68 \%, P<0.001)$. However, the FRS only predicted a marginally higher CAD risk (less than 1\%) in Scotland (12.5 \pm 10.5 vs.12.6 \pm 10.6 , $P=0.03$ ). Likewise, the overall number of genome-wide significant variants affecting CAD risk (157.6 \pm 7.7 and $157.5 \pm 7.7 ; P=0.12)$ and a wGRS for $C A D(2.49 \pm 0.25$ in both populations, $P=0.14)$ were remarkably similar in the English and Scottish population. Interestingly, we observed substantial differences in the allele frequencies of individual risk variants. Of the previously described 163 genome-wide significant variants studied here, 35 variants had higher frequencies in Scotland, whereas 37 had higher frequencies in England ( $P<0.001$ each).

Conclusions: Neither the traditional risk factors included in the FRS nor a genetic risk score (GRS) based on established common risk alleles explained the higher CAD prevalence in Scotland. However, we observed marked differences in the distribution of individual risk alleles, which emphasizes that even geographically and ethnically closely related populations may display relevant differences in the genetic architecture of a common disease.
\end{abstract}

Keywords: Coronary artery disease, Prevalence, Genetic risk score, UK Biobank

\section{Introduction}

CAD is one of the most prevalent complex diseases [1]. Its pathogenesis is influenced by an interplay of genetics, diet, lifestyle, environmental and socioeconomic factors [2]. Regional differences in CAD prevalence have been

\footnotetext{
*Correspondence: schunkert@dhm.mhn.de

1 Department of Cardiology, Deutsches Herzzentrum München,

Technische Universität München, Munich, Germany

Full list of author information is available at the end of the article
}

observed globally, continentally and even among adjacent countries. For example, as compared to the Netherlands and the United Kingdom (UK), Spain had a constantly lower CAD rate throughout the past 20 years [3]. The same phenomenon can be observed among populations within the UK. In the last 15 years Scotland had constantly higher CAD prevalence compared to England, Wales, and Northern Ireland-the underlying reasons being largely unclear [4]. 
In principle, both environmental factors or genetics may contribute to the local disparities in CAD $[5,6]$. Compared to the European population, Burokiene et al. found that high BMI and poor plasma lipid profiles are primarily responsible for higher cardiovascular disease (CVD) mortality in Lithuania whereas no difference was found for a genetic risk score based on 60 CVDassociated Single-nucleotide polymorphisms (SNPs) [7]. Indeed, exogenous risk factors affected by culture, lifestyle, or socioeconomics can undergo rapid changes on the individual, familial, and population level leading to marked temporal changes in CAD prevalence $[6,8]$.

Evolutionary genetics determine the allele frequency in a population, which is modulated by natural selection and stochastic forces such as genet drift [9]. These and other factors contribute to variation among individuals in the same population and across populations [8-10]. While mutations causing monogenic disorders are under evolutionary pressure, this applies, to a lesser extent to, common risk alleles with small effect sizes [10]. Indeed, genome-wide association studies (GWAS) revealed that most common cardiometabolic conditions like hypertension, diabetes mellitus, or hyperlipidemia are affected by hundreds of risk alleles, most of which are common [11]. The high number of susceptibility variants and their high allele frequencies jointly contribute to the genetic architecture of disease $[9,12]$.

Lately, genetic risk scoring has been found to be useful in CAD risk prediction as well as therapeutic and lifestyle guidance. Using a GRS based on 27 SNPs, Mega et al. observed that individuals at high genetic risk have greater benefit from statin therapy [13]. Moreover, Khera et al. showed that a healthy lifestyle drastically reduces risk of incident CAD events among individuals at high genetic risk [14]. Besides for individual disease risk prediction, GRS are also used to assess and compare the risk allele burden between populations with different disease prevalence. Keaton et al. found ethnic-specific differences in the genetic architecture in the context of type 2 diabetes (T2D) between African- and European-Americans [15], whereas Werissa et al. found no such difference between the Roma and the Hungarian general population [16]. Pima Indians in Arizona have the highest prevalence and incidence of non-insulin-dependent diabetes of any geographically defined population [17], but Hanson et al. found that this is not attributable to allele frequency differences at 63 diabetes loci [18].

In this study, we explored whether the higher CAD prevalence in the Scottish population could be explained by traditional risk factors and / or common genetic variants. We used a traditional scoring model, the FRS, and a GRS model based on 163 established common risk alleles.

\section{Materials and methods UK Biobank population}

UK Biobank (https://www.ukbiobank.ac.uk/) is a powerful prospective cohort study resource of $\sim 500,000$ volunteer participants originating from Great Britain. Genome-wide genotyping and various phenotypic data are available on nearly every participant [19]. At recruitment, participants answered a series of questions on lifestyle, health-related information and socio-demographics, and received a range of physical measures, which can be obtained by researchers. After quality control including filtering for sex discordance, missingness, heterozygosity, kinship coefficient and ethnic background, our study contained 442,860 individuals with eligible genotype data. Based on their country of birth, they were grouped into England, Northern Ireland, Wales, and Scotland (Additional file 1: Fig. 1, Table 1).

Baseline characteristics were recorded in the assessment centers of UK Biobank, such as age, gender, BMI, SBP, HDL-C, TC, and smoking status. Lifestyle and environment factors, as well as family history and ethnic background were self-reported. Medications and treatments were collected by questioning. To include more samples, we combined the UK Biobank data fields 20,003, 6177 and 6153 to extract antihypertensive drugs for each individual and used the same strategy to identify CAD and diabetes. All variables used in the FRS are available in Additional file 1: Table 2. All variables used in the QRISK 3 score are available in Additional file 1: Table 3. The diagnosis codes used to identify cases and the medication codes used to identify antihypertensives can be found in Additional file 1: Tables 4 and 5.

In order to keep measured factors consistent with age (reported at the first visit) for Framingham risk score calculation, we used only first recorded value (instance 0 data) from UK Biobank, at which participants were recruited from 2006 to 2010. The CAD prevalence and sample size can be found in Additional file 1: Table 1, and 371,077 individuals had both complete phenotype data and eligible genotype data (Additional file 1: Fig. 1, Table 6).

The study was approved by the Research Tissue Bank (RTB) and the National Research Ethics Service and UK Biobank's governing Research Ethics Committee (REC), and was conducted in accordance with the principles of the UK Biobank Ethics Advisory Committee (EAC).

\section{Source of CAD-associated SNPs}

Based on a review by Erdmann et al., we extracted lead SNPs of 163 CAD risk loci with genome-wide significance as derived from the decade of GWAS [20]. All 163 CAD-associated SNPs had odds ratios $>1.03$ (Additional file 1: Table 7) and were based on different individual 
studies using genotype data on 4,000,000 variants of more than 100,000 individuals. Besides, Khera et al. generated a CAD polygenetic risk score (PRS) including 6.6 million common genetic variants using a Bayesian approach called the LDpred algorithm, which uses an linkage disequilibrium (LD) reference panel to infer correlation patterns between SNPs for PRS calculations [21]. This genome-wide CAD PRS has more robust $P$ values and higher effect estimates on CAD prevalent and incidence [22].

\section{Calculation of the Framingham risk score and QRISK3 score} From the perspective of mathematical modeling, the Framingham risk prediction algorithm was developed and validated in large community-based samples, and the score is calculated by summing up all risk factors weighted by their estimated regression coefficients from Cox proportional hazards models for women and men separately [23]. The variables required to estimate the 10-year CVD risk include age, gender, SBP, HDL-C, TC, antihypertensive use, smoking status, and diabetes status, which are all available in UKB datasets. There are two models to calculate the FRS. The primary one uses lipids (HDL-C and TC), and the simpler one uses the BMI instead. The codes used to calculate the FRS_lipids and FRS_BMI can be found in Additional file 1: Table 10.

Hippisley-Cox et al. have developed the QRISK3 prediction algorithm that underlies 10-year cardiovascular disease risk in men and women [24]. The primary care systems presently recommend to prescribe statins to individuals with a QRISK3 risk score more than or equal to $10 \%$, according to the current guidelines in Wales and England [25, 26]. Therefore, we consider the QRISK3 score as an alternative to estimate the CAD risk. We included 199,778 individuals without missing data in any of the 22 QRISK3 variables, born in England and Scotland with genotype data (Additional file 1: Tables 3, 8). We used R Package QRISK3 (version 0.3.0) [24, 27] to calculate the 10-year CVD risk score for each individual. All 22 variables used in QRISK3 algorithm were available in UK Biobank Assessment Centre. Lifestyle, environment and family history were self-report, such as smoking and ethnic background. Medication and treatment were collected by verbal interview, such as corticosteroid use and antihypertensives treatment.

\section{Computation of $u$ GRS and wGRS}

After the exclusion of individuals with any missing phenotype data and genotype data of poor quality, we calculated the unweighted (uGRS, the raw counts or the number of risk alleles) and weighted (wGRS) genetic risk scores to assess whether the genetic risk at population level is different between England and Scotland.

$$
G R S=\sum_{k=1}^{K} b_{k} X_{k}
$$

In the SNP-based additive polygenic genetic model [28], Eq. (1), let $X_{1}, \ldots, X_{k}$ denotes the number of risk alleles of SNP $k$ in one individual, and let $b_{1}, \ldots, b_{k}$ denote the weight of SNP k. $X_{k}=0$ indicates no risk allele, while heterozygotes for the risk allele were coded as genotype $X_{k}=1$ and homozygotes for the risk allele as genotype $X_{k}=2$. Missing genotypes were imputed by their expected value, which is twice of the risk allele frequency in the population. Therefore, the effects of risk alleles at all loci are regarded as the same if all $b_{k}$ equal 1 . In this case a person's summary genetic risk score is the sum of all risk alleles at all loci, which is denoted as uGRS. Giving distinct weights to risk alleles of each SNP, alleles with larger effect size contribute more to the GRS, and wGRS is the sum of the number of risk alleles multiplied the corresponding log odds ratio of each risk allele. Additional file 1: Table 7 indicates the SNPs and risk alleles identified in independent GWAS studies [20], which are used for the uGRS as well as the log odds ratios for the wGRS.

\section{Statistical methods}

We used Pearson's chi-squared test to determine the significance of the difference in CAD prevalence between the two populations. The difference between two populations in FRS and QRISK3 were tested by two tail Mann-Whitney test as both are skewed and not normal distributions. The difference of means of the number of risk alleles and wGRS were assessed by two tail t-test as both are approximately normally distributed. Their distribution comparison was assessed by the KolmogorovSmirnov test. We used $\mathrm{R}$ version 4.0.3 with packages such as data.table [29], epiR, ggplot2, Table 1, and tidyverse for data analysis and plotting. PLINK2 was used to calculate uGRS and wGRS. PRSice-2 (Polygenic Risk Score Software for Biobank-Scale Data) was used to calculate a wGRS derived from 6.6 million variants [22]. The difference of risk alleles frequencies (RAF) between two populations are tested by Pearson's chi-squared test, and we adjust these $p$-values for multiple comparisons by Bonferroni correction. We used a significance level of $P<0.05$ for the means and distribution tests.

\section{Results}

\section{Baseline characteristics of study participants}

After exclusion of participants with missing covariates required for calculation of GRS or FRS, we obtained a set of 371,077 samples fulfilling our study requirements. The prevalence of CAD within UKB was highest in Scotland, 
Table 1 Basic Characteristics of participants born in England and Scotland in UK Biobank

\begin{tabular}{|c|c|c|c|}
\hline & England $(\mathrm{N}=317,889)$ & Scotland $(N=31,963)$ & $P$-value \\
\hline Gender*** & & & 0.0002 \\
\hline $\mathrm{F}$ & $169,679(53.4 \%)$ & $17,411(54.5 \%)$ & \\
\hline M & $148,210(46.6 \%)$ & $14,552(45.5 \%)$ & \\
\hline Age (years) & & & 0.0519 \\
\hline Mean (SD) & $56.7( \pm 8.1)$ & $56.6( \pm 8.0)$ & \\
\hline$B M I^{* * *}$ & & & 0.0002 \\
\hline Mean (SD) & $27.4( \pm 4.7)$ & $27.49( \pm 4.7)$ & \\
\hline HDL cholesterol (mg/dL) & & & 0.1296 \\
\hline Mean (SD) & $26.2( \pm 6.9)$ & $26.1( \pm 6.9)$ & \\
\hline Total cholesterol (mg/dL)* & & & 0.0381 \\
\hline Mean (SD) & $102.8( \pm 20.6)$ & $103.1( \pm 20.7)$ & \\
\hline Systolic blood pressure $(\mathrm{mmHg})^{* * *}$ & & & $<0.001$ \\
\hline Mean (SD) & $138.0( \pm 18.5)$ & $139.0( \pm 18.9)$ & \\
\hline Antihypertensive medication** & & & 0.0012 \\
\hline Yes & $72,816(22.9 \%)$ & $7,577(23.7 \%)$ & \\
\hline No & $245,073(77.1 \%)$ & $24,386(76.3 \%)$ & \\
\hline Smoking*** & & & $<0.001$ \\
\hline Yes & $32,484(10.2 \%)$ & $4,078(12.8 \%)$ & \\
\hline No & $285,405(89.8 \%)$ & $27,885(87.2 \%)$ & \\
\hline Diabetes*** & & & $<0.001$ \\
\hline Yes & $24,646(7.8 \%)$ & $1,918(6.0 \%)$ & \\
\hline No & $293,243(92.3 \%)$ & $30,045(94.0 \%)$ & \\
\hline
\end{tabular}

SD: standard deviation; BMI: body mass index; HDL: high-density lipoprotein

${ }^{*} p<0.05 ;{ }^{* *} p<0.01 ;{ }^{* * *} p<0.001$

followed by Wales, Northern Ireland, and England, which matches respective trends in published data from 2008 to 2010 [4] (Fig. 1, Additional file 1: Table 6). After data filtering, a significant difference in CAD prevalence was observed between England $(\mathrm{n}=317,889 ; 7.68 \%)$ and Scotland ( $\mathrm{n}=31,963 ; 8.98 \%, P<0.001)$, as well as between England and Wales $(\mathrm{n}=18,724 ; 8.30 \%, P=0.002)$, while there was no significant difference between England and Northern Ireland $(\mathrm{n}=2,501 ; 8.36 \%, P=0.20)$. Considering the well-established difference in CAD prevalence between Scotland and England, we focused our comparison on these two populations.

The English and Scottish participants had a similar mean age (56.7 \pm 8.1 in England and $56.6 \pm 8.0$ in Scotland) (Table 1). Among traditional CAD risk factors, the Scottish had moderate, but significantly higher levels of BMI and SBP $(P<0.001)$. There were also more smokers, but less diabetics among the Scottish compared to the English population $(P<0.001)$ (Table 1$)$.

\section{Comparison of traditional risk factors by the Framingham risk score and QRISK3 score}

FRS_lipidsranged from 0.5 to 94.7 (mean, $12.6 \pm 10.6$ ) for the Scottish, and from 0.3 to 96.1 (mean, $12.5 \pm 10.5$ ) for the English population ( $P=0.009$; Table 2, Fig. 2). Thus, the FRS explains a difference of CAD prevalence of less than $1 \%$ whereas the observed prevalence differed by $16.9 \%$ between the two countries. Computing the FRS BMI instead of lipids yielded similar results (Additional file 1: Fig. 2, Table 9). Likewise, estimation of CAD risk based on QRISK3 revealed only small but statistically significant differences between the two countries (Additional file 1: Fig. 3, Table 9).

\section{Comparison of the genetic burden by the polygenic risk score}

To investigate whether common genetic variants might predict the higher CAD prevalence in Scotland, we compared the population-based CAD GRS of Scotland and England based on 163 GWAS SNPs significantly associated with CAD (GWAS P $<5 \mathrm{E}-8$, OR $>1.03$ ) [20]. On average, Scottish participants had $157.5 \pm 7.7$ risk alleles while English individuals had 157.6 \pm 7.7 (Fig. 3, Table 2). Both, mean and distribution of uGRS based on 163 SNPs showed no significant difference between the two countries (Table 2). The same result was observed for wGRS based on CAD-associated SNPs. Namely, both countries had a mean wGRS of 10.6 and no difference in 


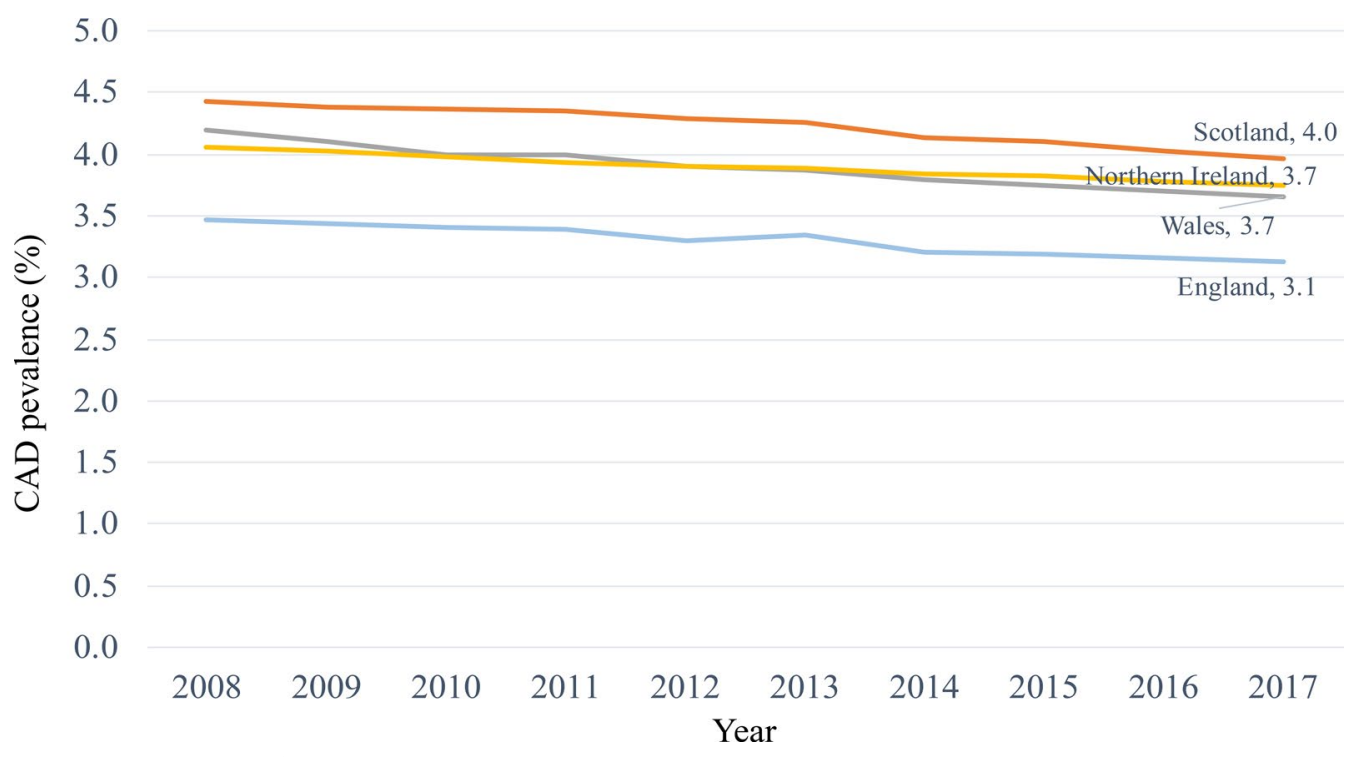

Fig. 1 Trends in the CAD prevalence from QOF data, England, Wales, Scotland, and Northern Ireland 2008 to 2017. Source: England—Health and Social Care Information Centre. QOF achievement data; Scotland —ISD Scotland. QOF achievement data to 2015/16. Prevalence data for 2016/17 and 2017/18 obtained via personal communication; Wales - StatsWales. QOF achievement data; Northern Ireland —Department of Health, Social Services and Public Safety. QOF exception reporting data 2017/18; QOF, Quality and Outcomes Framework [4]

Table 2 Statistics for the Framingham score and genetic risk score in populations

\begin{tabular}{llll}
\hline & England $(\mathbf{N}=\mathbf{3 1 7 , 8 8 9 )}$ & Scotland $(\mathbf{N}=\mathbf{3 1 , 9 6 3 )}$ & $\boldsymbol{P}$-value \\
\hline Framingham score using lipids** & & \\
Mean (SD) & $12.5(10.5)$ & $12.6(10.6)$ & 0.009 \\
Median [Min, Max] & $9.2[0.3,96.1]$ & $9.4[0.5,94.7]$ \\
Coronary artery disease uGRS & & \\
Mean (SD) & $157.6(7.7)$ & $157.5(7.7)$ \\
Median [Min, Max] & $157.6[122.1,196.0]$ & $158.0[122.6,186.7]$ \\
Coronary artery disease WGRS & $10.6(0.5)$ & $10.6(0.5)$ \\
Mean (SD) & $10.6[8.0,13.0]$ & $10.6[8.0,12.7]$ \\
Median [Min, Max] & & 0.1173 \\
\hline
\end{tabular}

SD: standard deviation

** $p<0.01$

wGRS distribution of the two populations was observable (Table 2, Additional file 1: Fig. 4). Finally, CAD risk based on a GRS derived from 6.6 million variants [21]22 revealed no differences between the two countries (Additional file 1: Fig. 5, Table 9).

\section{Comparison of risk allele frequencies}

We next calculated the risk allele frequency (RAF) at 163 loci with established genome-wide significant association with CAD in England and Scotland (Fig. 4, Additional file 1: Fig. 6, Table 7). There were 35 variants with higher RAF in Scotland whereas 37 had higher RAF in England (Fig. 4, $\mathrm{P}_{\text {adjust }}<0.001$ each). The absolute difference in
RAF ranged from 0.3\% (rs116843064, England $=98.1 \%$, Scotland $=97.8 \%$ ) to $3.3 \%$ (rs579459, England $=21.0 \%$, Scotland $=17.6 \%$ ). As mentioned above, these differences neutralized each other since the GRS displayed no significant differences between the two countries.

\section{Discussion}

The prevalence of CAD is higher in Scotland than in England for largely unexplained reasons $[4,30]$. This observation was also evident in the UK Biobank participants studied here. The traditional risk factors included in the FRS hardly explained the difference in CAD prevalence between the two countries. Out of 163 genome-wide 


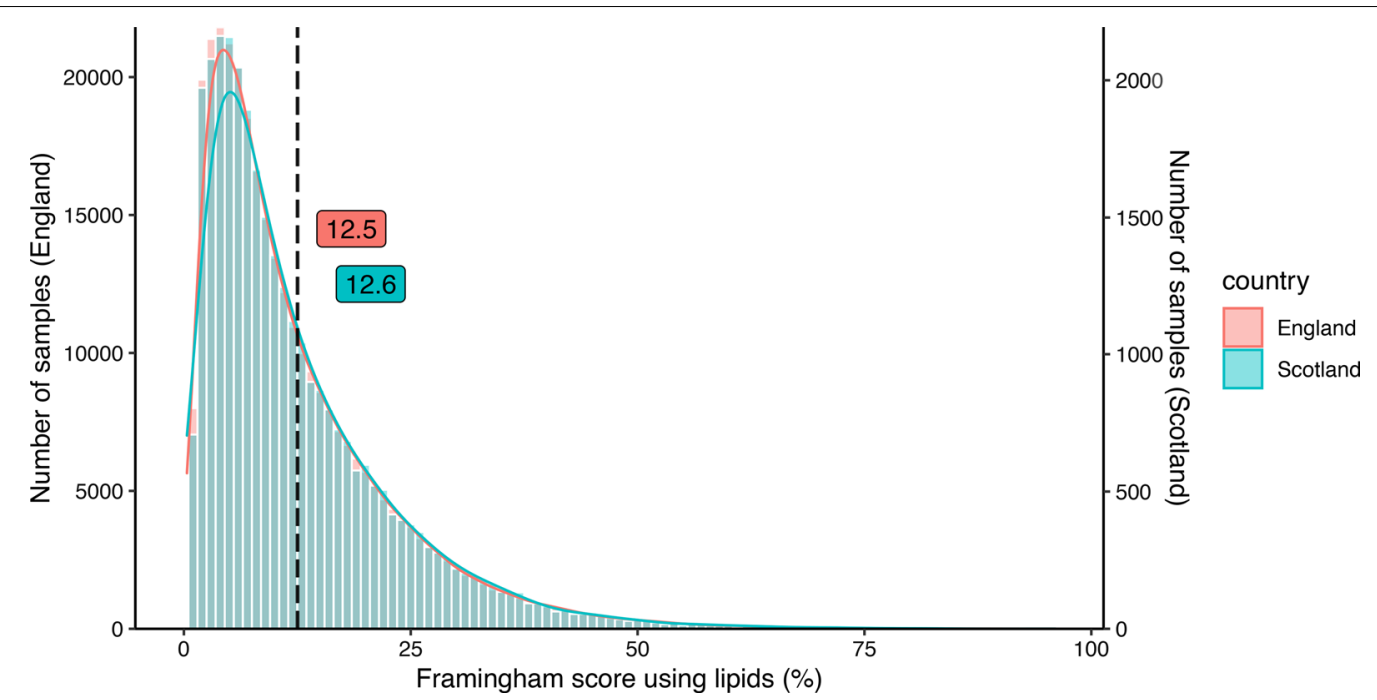

Fig. 2 Histograms showing the distribution of the Framingham score using lipids for the comparison population (born in Scotland) and the reference population (born in England)

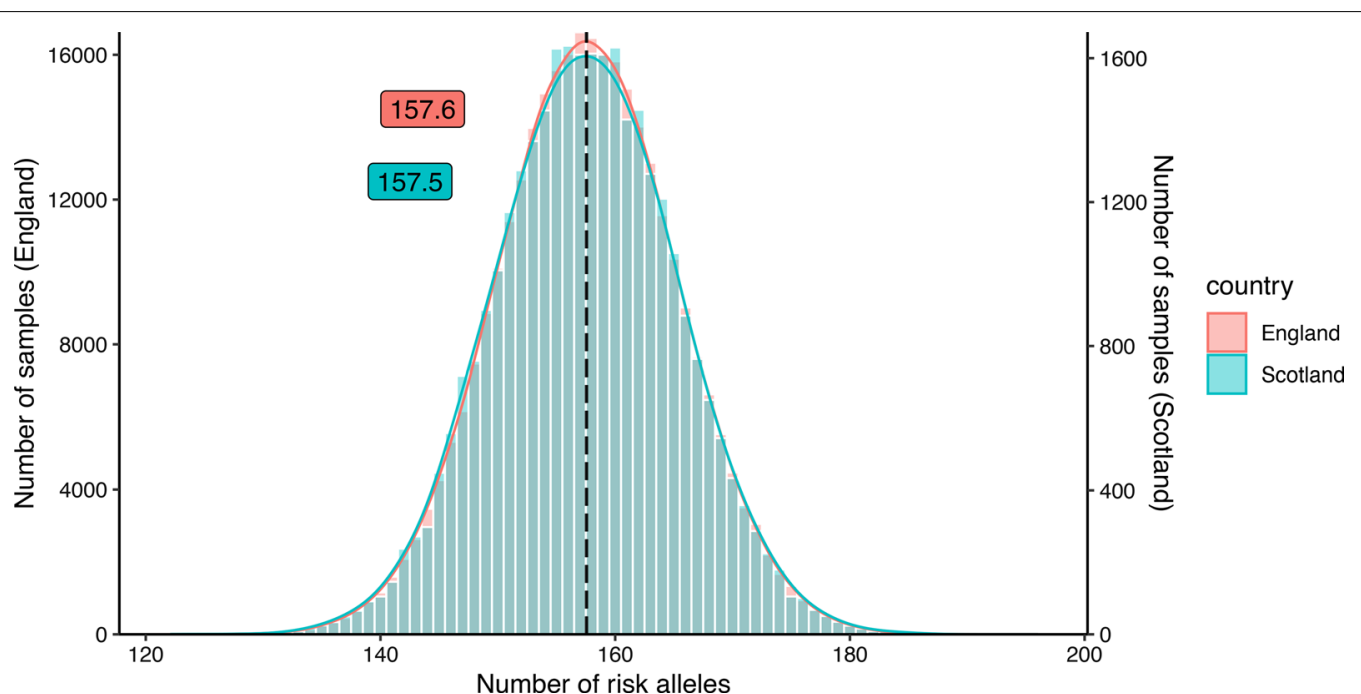

Fig. 3 Histograms showing the distribution of the number of risk alleles based on 163 CAD associated SNPs for the comparison population (born in Scotland) and the reference population (born in England)

significant risk alleles studied, 35 had higher RAF in Scotland whereas 37 had higher RAF in England. However, overall, these differences appeared to neutralize each other since there was no significant difference in the means and distributions of both weighted and unweighted GRS based on 163 CAD SNPs.

According to the ancestral-complex disease susceptibility model, genetic variations existed before the human spreading out of Africa and evolved with an extremely slow speed [31, 32]. However, nowadays environment and lifestyle are remarkably different from that of our ancestors. A mismatch between the ancestral variants and current environment might contribute to the development of some of non-communicable, complex diseases $[2,33]$.

It is unclear as to whether differences in ancestral variants contributing to CAD risk explain regional differences in CAD prevalence. With respect to England and Scotland, we observed that about $40 \%$ of genome-wide significant variants displayed significant differences in allele frequencies. It is remarkable to find that many significant differences in allele frequencies of disease 


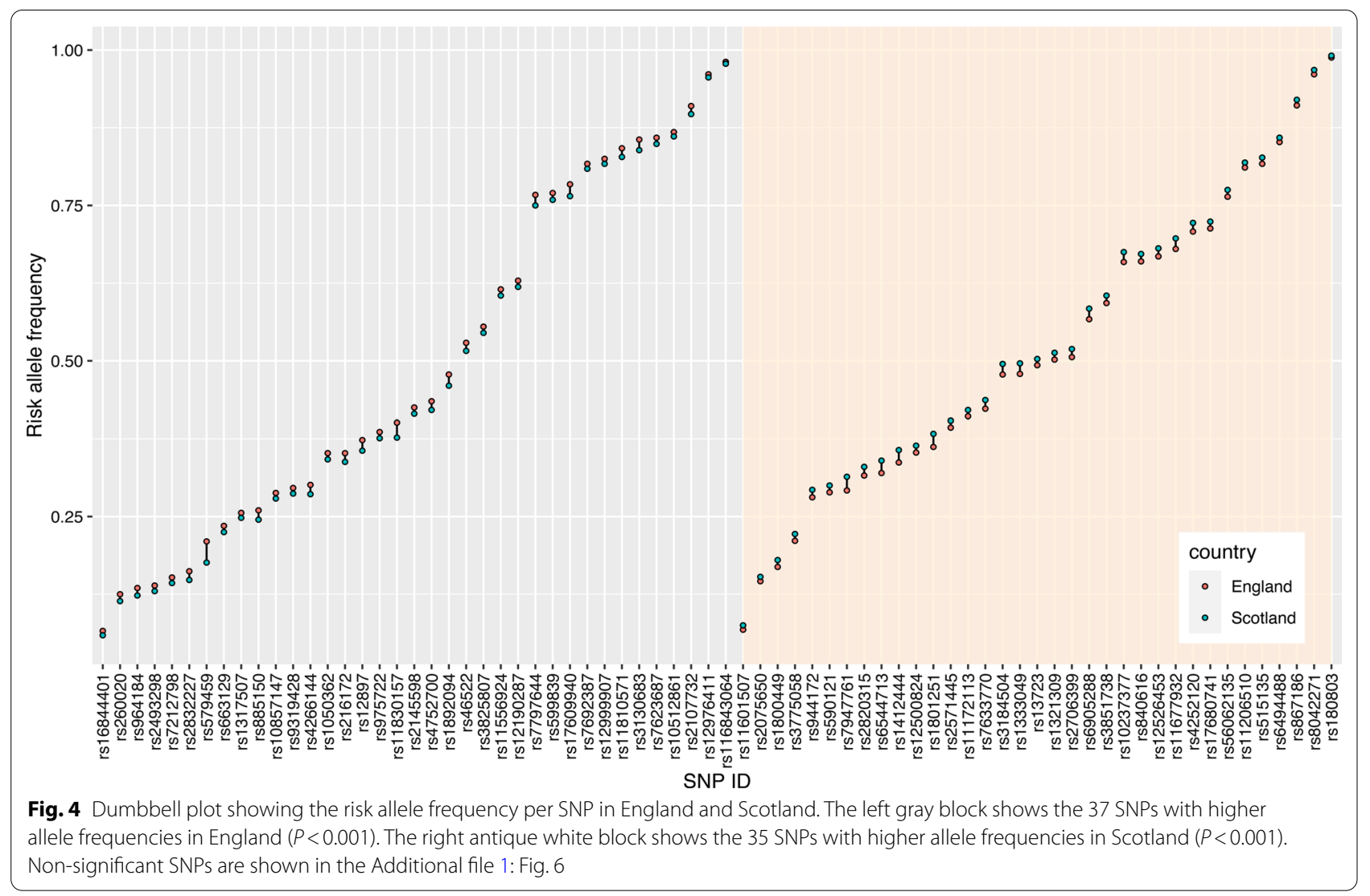

relevant genes in such closely related populations. However, the balanced effect-35 variants had higher RAF in Scotland and 37 had higher RAF in England-suggests that this is not driven by any selection pressure on these risk alleles, which is in line with findings of Keyue and Iftikhar, who did not observe significant differences in the distribution of Fst values at $158 \mathrm{CVD}$-associated SNPs compared to background SNPs [34]. In fact, the net effects of these differences at multiple loci seem to neutralize each other, since we observed no differences in the $\mathrm{CAD}$ risk based on polygenic risk scores.

Thus, genetic susceptibility to CAD-based to common risk alleles-appears to be rather similar in England and Scotland. The same applies to traditional risk factors for CAD, since the present as well as previous studies failed to demonstrate profound differences between these two countries [35, 36]. In 1989, Carstairs and Morris reported that Scotland suffers from more severe deprivation than England and Wales [37], In 2011, the same pattern of deprivation was still observed between the countries of Scotland and England [38]. In 2013, Newton et al. reported that significant health inequalities remain between the poorest and most deprived areas [39]. Thus, social deprivation might be one of the explanations for Scotland's higher CAD rates. In order to lower CAD rates in Scotland, it seems to be reasonable to intensify preventive measures to be delivered at the most deprived.

A limitation of our study may be the fact that the lead SNPs we used to represent risk at a given genome-wide significant locus might not be the causal ones. However, these variants were associated with the strongest risk such that the causal variants are likely to be in very high LD. Moreover, the estimation of risk based on polygenic risk scores is unlikely to be affected by lack of knowledge on the causal variant. Another limitation of our study could be that we did not explore rare variants, genegene interactions, gene-environment, and exposure to epigenetic factors. All of these can modulate genetic risk $[2,40,41]$ but are challenging to investigate in a study like ours. As for the traditional factors analysis, we only included the major risk factors for CAD (sex, age, BMI, HDL-C, TC, SBP, antihypertensive medication, smoking status and diabetes), while other important factors such as physical activity, family history and socioeconomic status are not included in the Framingham risk model [42]. Finally, the UKB population has been considered to represent a relatively low risk. As such, the data may not be representative for the entire population spectrum [43]. Nevertheless, the repeatedly observed differences 
in CAD prevalence between Scotland and England were apparent in UKB as well.

\section{Conclusions}

Using representative data from UK Biobank, our study assessed traditional and genetic risk models for discrimination of CAD prevalence in Scotland and England. Our study found that the traditional risk factors included in FRS may explain little of the difference in CAD prevalence between Scotland and England. Likewise, both unweighted and weighted GRS based on 163 SNPs or 6.6 million SNPs suggested a similar genetic susceptibility to CAD in the Scottish and English populations. Yet, there have to be reasons why the Scottish population constantly has higher CAD rates than the English. If genetics can't elucidate this observation, environmental or lifestyle factors that have received less attention thus far might provide an answer [44-47].

\begin{abstract}
Abbreviations
CAD: Coronary artery disease; UK: United Kingdom; FRS: Framingham risk score; GRS: Genetic risk score; UGRS: Unweighted genetic risk score; wGRS: Weighted genetic risk score; CVD: Cardiovascular disease; SNP: Single nucleotide polymorphism; GWAS: Genome-wide association studies; BMI: Body mass index; SBP: Systolic blood pressure; HDL-C: Low Density Lipoprotein-Cholesterin; LD: Linkage disequilibrium; PRS: Polygenetic risk score; TC: Total cholesterol; FRS_lipids: Framingham risk score using lipids; FRS_BMI: Framingham risk score using BMI; UKB: UK Biobank; RAF: Risk allele frequency.
\end{abstract}

\section{Supplementary Information}

The online version contains supplementary material available at https://doi. org/10.1186/s12872-021-02398-4.

Additional file 1. Flowcharts, data preparation, results and code, which are all designed, tidied and calculated by ourselves.

\section{Acknowledgements}

The present analyses were conducted using the UK Biobank resource under Application 25214. We thank the participants and investigators in this analysis.

\section{Authors' contributions}

H.S. conceived and designed the project, guided manuscript preparation and participated in manuscript drafting and writing. C.Y. analyzed data, performed the statistical analysis, figures plot and manuscript drafting and writing. F.S. gave conceptual advice in Framingham risk score and QRISK3 score calculation and participated in manuscript drafting. S.P. gave conceptual advice in GRS calculation and statistical methods and participated in manuscript drafting. Z.C., U.G. gave conceptual advice and participated in manuscript drafting. L.L gave conceptual advice. M.H gave conceptual advice and participated in manuscript drafting. All authors read and approved the final manuscript.

\section{Funding}

Open Access funding enabled and organized by Projekt DEAL. The work was funded by the German Federal Ministry of Education and Research (BMBF) within the framework of ERA-NET on Cardiovascular Disease (DruggableMl-genes: 01 KL1802), within the scheme of target validation (BlockCAD: 16GW0198K), and within the framework of the e:Med research and funding concept (AbCD-Net: 01ZX1706C). As a Co-applicant of the British Heart Foundation (BHF)/German Centre of Cardiovascular Research (DZHK)-collaboration (DZHK-BHF: 81X2600522) and the Leduca Foundation for Cardiovascular Research (PlaqOmics: 18CVD02), we gratefully acknowledge their funding.
Additional support has been received from the German Research Foundation (DFG) as part of the Sonderforschungsbereich SFB 1123 (B02) and the Sonderforschungsbereich SFB TRR 267 (B05). Further, we kindly acknowledge the support of the Bavarian State Ministry of Health and Care who funded this work with DigiMed Bayern (grant No: DMB-1805-0001) within its Masterplan "Bayern Digital II" and of the German Federal Ministry of Economics and Energy in its scheme of ModulMax (grant No: ZF4590201BA8). MH is grateful for support by the Federal Ministry of Education and Research grants 01ZX1408D and 01ZX1708G within the e:Med program. The funders had no role in the study design, data collection and analysis, decision to publish, or preparation of the manuscript.

\section{Availability of data and materials}

The datasets analyzed during the current study are available in UK Biobank: https://www.ukbiobank.ac.uk/. The codes used to calculate the FRS_lipids and FRS_BMI can be found in Additional file 1. R package for analysis: https://www. bioconductor.org/

\section{Declarations}

\section{Ethics approval and consent to participate}

UK Biobank has approval from the North West Multi-centre Research Ethics Committee (MREC), which covers the UK. It also sought the approval in England and Wales from the Patient Information Advisory Group (PIAG) for gaining access to information that would allow it to invite people to participate. PIAG has since been replaced by the National Information Governance Board for Health \& Social Care (NIGB). In Scotland, UK Biobank has approval from the Community Health Index Advisory Group (CHIAG).

\section{Consent for publication}

UK Biobank possesses a Human Tissue Authority (HTA) licence, so a separate HTA licence is not required by researchers who receive samples from the resource. Instead of requiring each applicant to obtain separate ethics approval, UK Biobank has sought generic Research Tissue Bank (RTB) approval, which should cover the large majority of research using the resource. This approach is recommended by the National Research Ethics Service and UK Biobank's governing Research Ethics Committee (REC), which approved the application in 2010.

\section{Competing interests}

The authors declare that there is no known competing financial interests or personal relationships that could have appeared to influence the work reported in this paper.

\section{Author details}

${ }^{1}$ Department of Cardiology, Deutsches Herzzentrum München, Technische Universität München, Munich, Germany. ${ }^{2}$ Deutsches Zentrum Für Herz- Und Kreislauferkrankungen (DZHK), Partner Site Munich Heart Alliance, Munich, Germany. ${ }^{3}$ Medical Graduate Center, Technische Universität München, Munich, Germany. ${ }^{4}$ Department of Informatics, Technische Universität München, Munich, Germany. ${ }^{5}$ Institute of Computational Biology ICB, Helmholtz Zentrum München (HMGU), Munich, Germany. ${ }^{6}$ German Heart Center Munich, Technical University Munich, Lazarettstraße 36, 80636 Munich, Germany.

Received: 11 September 2021 Accepted: 22 November 2021

Published online: 07 December 2021

\section{References}

1. Kessler T, Schunkert H. Coronary artery disease genetics enlightened by genome-wide association studies. JACC: Basic TransI Sci. 2021;6:610-23.

2. Gurdasani D, Barroso I, Zeggini E, Sandhu MS. Genomics of disease risk in globally diverse populations. Nat Rev Genet. 2019;20:520-35.

3. Timmis A, Townsend N, Gale CP, Torbica A, Lettino M, Petersen SE, et al. European society of cardiology: cardiovascular disease statistics 2019. Eur Heart J. 2020;41:12-85.

4. Bhatnagar P, Wickramasinghe K, Wilkins E, Townsend N. Trends in the epidemiology of cardiovascular disease in the UK. Heart. 2016;102:1945-52. 
5. Graham G. Disparities in cardiovascular disease risk in the United States. Curr Cardiol Rev. 2015;11:238-45.

6. Peasey A, Bobak M, Kubinova R, Malyutina S, Pajak A, Tamosiunas A, et al. Determinants of cardiovascular disease and other non-communicable diseases in Central and Eastern Europe: Rationale and design of the HAPIEE study. BMC Public Health. 2006;6:255.

7. Burokienè N, Domarkienè I, Ambrozaityte L, Uktverytè I, Meškienè R, Karčiauskaite $D$, et al. Classical rather than genetic risk factors account for high cardiovascular disease prevalence in Lithuania: a cross-sectional population study. Adv Med Sci. 2017;62:121-8.

8. Kessler T, Vilne B, Schunkert H. The impact of genome-wide association studies on the pathophysiology and therapy of cardiovascular disease. EMBO Mol Med. 2016;8:688-701.

9. Ding K, Kullo IJ. Evolutionary genetics of coronary heart disease. Circulation. 2009;1 19:459-67.

10. Chen Z, Schunkert H. Genetics of coronary artery disease in the postGWAS era. J Internal Med. 2021. https://doi.org/10.1111/joim.13362.

11. Tam V, Patel N, Turcotte M, Bossé Y, Paré G, Meyre D. Benefits and limitations of genome-wide association studies. Nat Rev Genet. 2019:20:467-84.

12. Wray NR, Goddard ME, Visscher PM. Prediction of individual genetic risk to disease from genome-wide association studies. Genome Res 2007;17:1520-8

13. Mega JL, Stitziel NO, Smith JG, Chasman DI, Caulfield M, Devlin JJ, et al. Genetic risk, coronary heart disease events, and the clinical benefit of statin therapy: an analysis of primary and secondary prevention trials. Lancet. 2015:385:2264-71.

14. Khera AV, Emdin CA, Drake I, Natarajan P, Bick AG, Cook NR, et al. Genetic risk, adherence to a healthy lifestyle, and coronary disease. N Engl J Med. 2016;375:2349-58.

15. Keaton JM, Bailey JNC, Palmer ND, Freedman BI, Langefeld CD, Ng MC et al. A comparison of type 2 diabetes risk allele load between African Americans and European Americans. Hum Genet. 2014;133:1487-95.

16. Werissa NA, Piko P, Fiatal S, Kosa Z, Sandor J, Adany R. SNP-based genetic risk score modeling suggests no increased genetic susceptibility of the Roma population to type 2 diabetes mellitus. Genes. 2019;10:942.

17. Knowler WC, Pettitt DJ, Saad MF, Bennett PH. Diabetes mellitus in the Pima Indians: incidence, risk factors and pathogenesis. Diabetes Metab Rev. 1990;6:1-27.

18. Hanson RL, Rong R, Kobes S, Muller YL, Weil EJ, Curtis JM, et al. Role of established type 2 diabetes-susceptibility genetic variants in a high prevalence American Indian population. Diabetes. 2015;64:2646-57.

19. Bycroft C, Freeman C, Petkova D, Band G, Elliott LT, Sharp K, et al. The UK Biobank resource with deep phenotyping and genomic data. Nature 2018;562:203-9.

20. Erdmann J, Kessler T, Munoz Venegas L, Schunkert H. A decade of genome-wide association studies for coronary artery disease: the challenges ahead. Cardiovasc Res. 2018;114:1241-57.

21. Vilhjálmsson BJ, Yang J, Finucane HK, Gusev A, Lindström S, Ripke S, et al. Modeling linkage disequilibrium increases accuracy of polygenic risk scores. Am J Hum Genet. 2015:97:576-92.

22. Aragam KG, Natarajan P. Polygenic scores to assess atherosclerotic cardiovascular disease risk. Circ Res. 2020;126:1159-77.

23. D'Agostino RB, Vasan RS, Pencina MJ, Wolf PA, Cobain M, Massaro JM, et al. General cardiovascular risk profile for use in primary care: the Framingham Heart Study. Circulation. 2008;117:743-53.

24. Hippisley-Cox J, Coupland C, Brindle P. Development and validation of QRISK3 risk prediction algorithms to estimate future risk of cardiovascular disease: prospective cohort study. BMJ. 2017;357:2099.

25. Cardiovascular disease: risk assessment and reduction, including lipid modification. London: National Institute for Health and Care Excellence (UK); 2016. http://www.ncbi.n/m.nih.gov/books/NBK554923/. Accessed 5 May 2021.

26. Duerden M, O'Flynn N, Qureshi N. Cardiovascular risk assessment and lipid modification: NICE guideline. Br J Gen Pract. 2015;65:378-80.

27. LiY, Sperrin M, van Staa T. R package "QRISK3": an unofficial research purposed implementation of ClinRisk's QRISK3 algorithm into R. F1000Res. 2020:8:2139.

28. International Schizophrenia Consortium, Purcell SM, Wray NR, Stone JL, Visscher PM, O'Donovan MC, et al. Common polygenic variation contributes to risk of schizophrenia and bipolar disorder. Nature. 2009;460:748-52.

29. Dowle M, Srinivasan A, Gorecki J, Chirico M, Stetsenko P, Short T, et al. Package 'data. table.' Extension of 'data frame. 2019.

30. Heart \& Circulatory Disease Statistics 2019. https://www.bhf.org.uk/whatwe-do/our-research/heart-statistics/heart-statistics-publications/cardi ovascular-disease-statistics-2019. Accessed 5 May 2021.

31. Van Den Biggelaar AHJ, De Craen AJM, Gussekloo J, Huizinga TWJ, Heijmans BT, Frölich M, et al. Inflammation underlying cardiovascular mortality is a late consequence of evolutionary programming. FASEB $J$. 2004;18:1022-4.

32. Di Rienzo A, Hudson RR. An evolutionary framework for common diseases: the ancestral-susceptibility model. Trends Genet. 2005;21:596-601.

33. Bhatnagar A. Environmental determinants of cardiovascular disease. Circ Res. 2017;121:162-80.

34. Ding K, Kullo IJ. Geographic differences in allele frequencies of susceptibility SNPs for cardiovascular disease. BMC Med Genet. 2011;12:55.

35. Brindle PM, McConnachie A, Upton MN, Hart CL, Smith GD, Watt GC. The accuracy of the Framingham risk-score in different socioeconomic groups: a prospective study. Br J Gen Pract. 2005;55:838-45.

36. Tunstall-Pedoe H, Woodward M. SIGN group on risk estimation. By neglecting deprivation, cardiovascular risk scoring will exacerbate social gradients in disease. Heart. 2006;92:307-10.

37. Carstairs V, Morris R. Deprivation: explaining differences in mortality between Scotland and England and Wales. BMJ. 1989;299:886-9.

38. Yousaf S, Bonsall A. UK Townsend Deprivation Scores from 2011 census data. Colchester: UK Data Service; 2017

39. Newton JN, Briggs ADM, Murray CJL, Dicker D, Foreman KJ, Wang H, et al. Changes in health in England, with analysis by English regions and areas of deprivation, 1990-2013: a systematic analysis for the Global Burden of Disease Study 2013. The Lancet. 2015;386:2257-74.

40. Khera AV, Kathiresan S. Genetics of coronary artery disease: discovery, biology and clinical translation. Nat Rev Genet. 2017;18:331-44.

41. Musunuru K, Kathiresan S. Genetics of common, complex coronary artery disease. Cell. 2019;177:132-45.

42. Malakar AK, Choudhury D, Halder B, Paul P, Uddin A, Chakraborty S. A review on coronary artery disease, its risk factors, and therapeutics. J Cell Physiol. 2019;234:16812-23.

43. Stamatakis E, Owen KB, Shepherd L, Drayton B, Hamer M, Bauman AE. Is cohort representativeness Passé? Poststratified associations of lifestyle risk factors with mortality in the UK Biobank. Epidemiology. 2021:32:179-88.

44. Holmes SD, Krantz DS, Rogers H, Gottdiener J, Contrada RJ. Mental stress and coronary artery disease: a multidisciplinary guide. Prog Cardiovasc Dis. 2006:49:106-22.

45. Grazuleviciene R, Vencloviene J, Kubilius R, Grizas V, Danileviciute A, Dedele A, et al. Tracking restoration of park and urban street settings in coronary artery disease patients. Int J Environ Res Public Health. 2016;13:550.

46. Kazemian N, Mahmoudi M, Halperin F, Wu JC, Pakpour S. Gut microbiota and cardiovascular disease: opportunities and challenges. Microbiome. 2020;8:36.

47. Turgeon PJ, Sukumar AN, Marsden PA. Epigenetics of cardiovascular disease: a new 'beat' in coronary artery disease. MEE. 2014;2:37-52.

\section{Publisher's Note}

Springer Nature remains neutral with regard to jurisdictional claims in published maps and institutional affiliations. 